\title{
Aplicação da teoria de médio alcance para enfermagem em reabilitação cardiovascular de pacientes com insuficiência cardíaca
}

Application of a middle-range nursing theory for cardiovascular rehabilitation of patients with heart failure

Aplicación de una teoría de medio alcance en enfermería para rehabilitación cardiovascular de pacientes con insuficiencia cardiaca

\section{Resumo}

Este estudo teve por objetivo descrever como a teoria de médio alcance para enfermagem em reabilitação cardiovascular, a partir da utilização do processo de enfermagem, contribuiu para o processo de reabilitação cardiovascular em pacientes com insuficiência cardíaca. Realizou-se um estudo quase experimental, não randomizado, baseado em pré e pós-intervenção. A amostra foi constituída por 16 pacientes internados em uma unidade de referência em cuidados com insuficiência cardíaca. Alcançou-se um total de 14 diagnósticos prioritários para os respectivos participantes. Os diagnósticos mais incidentes foram ansiedade, medo, intolerância à atividade e fadiga. Pode-se concluir que a assistência à luz da teoria de médio alcance para enfermagem em reabilitação cardiovascular, favorece um adequado processo de reabilitação à pacientes com Insuficiência cardíaca, pois houve uma redução na manifestação de diagnósticos prioritários, em pacientes com insuficiência cardíaca, permitindo assim mais conforto e qualidade de vida, o que é essencial para o processo de reabilitação cardiovascular.

Palavras-chave: Enfermagem; Teoria de enfermagem; Reabilitação cardíaca; Adaptação.

\begin{abstract}
This study aimed to describe how the middle-range theory for nursing in cardiovascular rehabilitation, based on the use of the nursing process, contributed to the cardiovascular rehabilitation process in patients with heart failure. A quasi-experimental, non-randomized study was performed, using a pre- and post-intervention approach. The sample consisted of 16 patients hospitalized in a referral unit for the treatment of heart failure. A total of 14 priority nursing diagnoses were identified for the participants. The most incident nursing diagnoses were anxiety, fear, activity intolerance and fatigue. It can be concluded that assistance in the light of the middle-range theory for nursing in cardiovascular rehabilitation favors an adequate rehabilitation process for patients with heart failure, as there was a reduction in the manifestation of priority diagnoses in patients with heart failure, thus allowing more comfort and quality of life, which are essential elements for the cardiovascular rehabilitation process.
\end{abstract}

Keywords: Nursing; Nursing theory; Cardiac rehabilitation; Adaptation. 


\begin{abstract}
Resumen
Este estudio tuvo como objetivo describir cómo la teoría de medio alcance para la enfermería en rehabilitación cardiovascular, basada en el uso del proceso de enfermería, contribuyó para el proceso de rehabilitación cardiovascular en pacientes con insuficiencia cardíaca. Se realizó un estudio cuasi-experimental, no aleatorizado, con abordaje pre y post-intervención. La muestra estuvo constituida por 16 pacientes hospitalizados en una unidad de referencia para el tratamiento de insuficiencia cardiaca. Se identificaron un total de 14 diagnósticos de enfermería prioritarios para los participantes. Los diagnósticos de enfermería más incidentes fueron ansiedad, miedo, intolerancia a la actividad y fatiga. Se puede concluir que la asistencia a la luz de la teoría de medio alcance para la enfermería en rehabilitación cardiovascular favorece un adecuado proceso de rehabilitación de pacientes con insuficiencia cardíaca, ya que hubo una reducción en la manifestación de diagnósticos prioritarios en pacientes con insuficiencia cardíaca, lo que permitió más confort y calidad de vida, elementos esenciales para el proceso de rehabilitación cardiovascular.
\end{abstract}

Palabras clave: Enfermería; Teoría de enfermería; Rehabilitación cardiaca; Adaptación.

\title{
1. Introdução
}

A Insuficiência Cardíaca (IC) pode ser definida como um desfecho clínico para inúmeros outros adoecimentos cardiovasculares, esta tem ganhado cada vez mais destaque, principalmente devido ao aumento da sua prevalência e alterações causadas na vida do ser portador. Trata-se de uma síndrome clínica complexa na qual o coração se torna incapaz de bombear sangue a fim de atender as necessidades metabólicas tissulares, ou faz com altas pressões de preenchimento (Sociedade Brasileira de Cardiologia, 2018).

Diante disto, ressalta-se a importância de trabalhar a reabilitação cardiovascular uma vez que o acometimento supracitado gera um quadro crônico que necessita de adaptação. Segundo a Organização Mundial da Saúde, a Reabilitação Cardiovascular (RCV) é o somatório das atividades necessárias para garantir aos pacientes, portadores de cardiopatia, as melhores condições física, mental e social, de forma que eles consigam, pelo seu próprio esforço, reconquistar uma posição normal na comunidade e levar uma vida ativa e produtiva (Brown, 1964).

Considera-se que a enfermagem é uma profissão em ascensão, com destaque para a pesquisa. Ao longo dos anos, tal ciência se desenvolveu bastante e de forma proporcional a necessidade de um cuidado mais específico, singular e preciso. Tais avanços possibilitaram inúmeras descobertas científicas principalmente no tocante do desenvolvimento de teorias que, por sua vez, favorece um cuidado mais direcionado e com embasamento científico.

É certo que os avanços da ciência e tecnologia contribuem, de forma direta, para uma melhor qualidade no cuidado à pessoa em adoecimento cardiovascular. No entanto, a busca pela melhoria da qualidade do cuidado não depende só dos avanços científicos e tecnológicos, mas também da utilização deste conhecimento pelos profissionais.

Neste contexto, os enfermeiros precisam utilizar-se de tecnologias como metodologia sistematizada e terminologias próprias para que o cuidado seja realizado de forma mais consistente. Para tanto, o Conselho Federal de Enfermagem editou a Resolução 358/2009 sobre uso de habilidades técnico-científicas que favorecem a organização e sistematização do cuidado, cuja utilização colabora na implementação do Processo de Enfermagem (PE). A referida Resolução defende que o PE deve-se embasar em uma teoria de enfermagem que oriente suas etapas de coleta de dados, diagnósticos de enfermagem, planejamento das intervenções/atividades e avaliação dos resultados alcançados (Conselho Federal de Enfermagem, 2009).

Portanto, o presente estudo fez uso da Teoria de Médio Alcance em Enfermagem para Reabilitação Cardiovascular (TMA-Enf RCV). A RCV foi considerada como um processo que envolve a implementação de intervenções dirigidas à pessoa pós-evento cardiovascular, com vistas à reabilitação física, social e psicológica, tornando-a capaz de manter as atividades cotidianas (Farias, 2018).

$\mathrm{Na}$ enfermagem, as teorias de médio alcance têm sido bastante utilizadas e apoiadas por diversos fatores, destaca-se a sua grande utilidade para as pesquisas e o seu baixo nível de abstração, o que as tornam mais facilmente aplicáveis na prática clínica (Leandro et al., 2018). Assim, surgiu a seguinte questão de investigação: A utilização da TMA-Enf RCV contribui para o processo de reabilitação cardiovascular em pacientes com insuficiência cardíaca? O objetivo deste estudo é descrever como a 
teoria de médio alcance para enfermagem em reabilitação cardiovascular, a partir da utilização do processo de enfermagem, contribuiu para o processo de reabilitação cardiovascular em pacientes com insuficiência cardíaca.

\section{Metodologia}

Realizou-se um estudo quase experimental, não randomizado, baseado em pré e pós-intervenção. O quase experimento é um tipo de delineamento que testa uma intervenção em que os participantes não são distribuídos randomicamente entre as condições de tratamento, também conhecido como experimento de campo, experimento in situ, ensaio não randomizado ou ensaio controlado sem randomização (Polit, 2019).

A pesquisa ocorreu em um hospital terciário do Sistema Único de Saúde (SUS) certificado pelo Ministério da Saúde e pelo Ministério da Educação como instituição de ensino, desenvolvendo atividades específicas no tocante à área cardíaca e pulmonar. A coleta e dados foi realizada em uma unidade de internação, tipo enfermaria, denominada I, que contém 28 leitos destinados a pacientes com IC, no período de setembro a janeiro de 2021. Utilizou-se amostragem por conveniência.

Estabeleceram-se como critérios de inclusão idade acima de 18 anos e ter IC classe funcional II ou III de acordo com a New York Heart Association (NYHA). Foram excluídos os pacientes em quadro agudo da doença e aqueles sem condições cognitivas ou físicas para interagir com o pesquisador e ser submetido ao Mini-Exame do Estado Mental.

O estudo foi constituído por 4 momentos distintos que foram desde a identificação da amostra, passando pelo levantamento das principais necessidades de reabilitação cardiovascular dos participantes, identificação dos diagnósticos de enfermagem e aplicação das intervenções, até a avaliação dos resultados obtidos. Os detalhes de cada momento podem ser mais bem visualizados na Figura 1.

Figura 1. Organização do percurso metodológico no desenvolvimento do estudo.

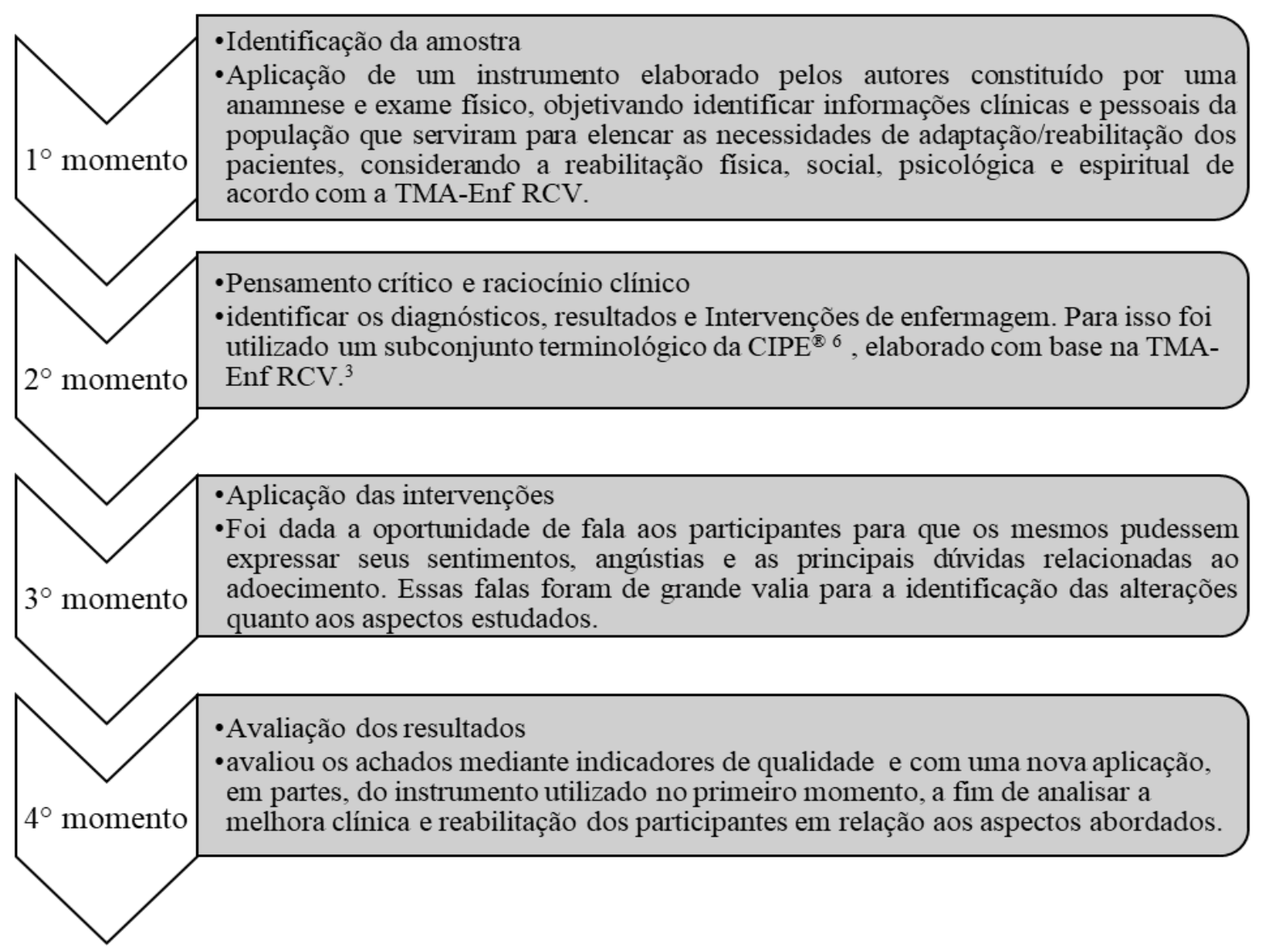

Fonte: Elaborado pelos autores. 
O projeto de pesquisa que deu origem ao presente estudo foi aprovado pelo Comitê Ética em Pesquisa do Hospital de Messejana Dr. Carlos Alberto Studart Gomes, obedecendo aos preceitos éticos da Resolução 466/2012 (parecer n ${ }^{\circ}$. 4.184.426, CAAE $n^{\circ}$. 34604620.0.0000.5039). Todos os participantes que concordaram em participar do estudo assinaram o Termo de Consentimento Livre e Esclarecido.

\section{Resultados}

O estudo contou com 16 participantes, predominantemente do sexo masculino (68,7\%), com média de idade acima dos 61 anos e renda de até 1 salário-mínimo, confirmando o que foi encontrado em outras pesquisas na literatura cujo foco foi pacientes com IC (Lima et al., 2019; Poffo et al., 2017; Xavier et al., 2020).

O processo de reabilitação cardiovascular de pacientes com insuficiência cardíaca necessita de uma visão ampla e abrangente, enganam-se os que focam seus cuidados apenas no aspecto físico, justificado pela incapacidade na realização das atividades básicas de seus pacientes.

Na sua totalidade, entende-se como reabilitação cardiovascular, um processo assistencial, árduo que pode contribuir de forma significante no controle e melhora do quadro clínico do paciente com adoecimento cardíaco, esta é ampla e atua a fim de suprir necessidades físicas, sociais, psicológicas e espirituais que os pacientes venham apresentar, buscando a resolubilidade das mesmas, favorecendo um retorno as condições ideais na medida do possível (Farias et al., 2021).

Após a etapa de pensamento crítico e raciocínio clínico, diante dos dados obtidos na anamnese e exame físico dos participantes, utilizou-se um subconjunto terminológico da CIPE®, permitindo assim a identificação total de 14 diagnósticos de enfermagem (Rolim, 2020). Estes foram organizados de acordo com cinco conceitos da TMA Enf-RCV, sendo eles: Cuidado reabilitador; Processo educativo; Apoio psicossocial ao paciente e família; Programa de reabilitação cardiovascular supervisionado e Terapia baseada no exercício. A tabela 1 mostra de forma simplificada os diagnósticos identificados nos participantes da pesquisa.

Tabela 1. Diagnósticos de enfermagem identificados conforme o subconjunto terminológico de Rolim (2020) relacionado aos conceitos da TMA-Enf RCV de Farias (2018) ( $n=16)$.

\begin{tabular}{ll}
\hline Conceitos & Diagnósticos de Enfermagem \\
\hline Cuidado Reabilitador & Desconforto \\
\hline Processo educativo & Sono e repouso prejudicado \\
\hline Apoio psicossocial ao paciente e família & Autocuidado prejudicado \\
& Atitude em relação ao manejo da medicação conflituosa \\
\hline Programa de reabilitação cardiovascular & Angústia espiritual \\
supervisionado & Baixa autoestima situacional \\
& Desesperança \\
& Medo \\
\hline Terapia baseada no exercício & Ansiedade \\
& Dispneia \\
& Edema \\
\hline
\end{tabular}

Fonte: Elaborado pelos autores. 
Dentre os cinco conceitos da teoria utilizada, destacaram-se com maior quantitativo de enunciados diagnósticos e intervenções de enfermagem validado "Apoio psicossocial ao paciente e à família" e "Programa de Reabilitação Cardiovascular Supervisionado". Para o conceito Apoio Psicossocial ao Paciente e à Família, os Diagnósticos de Enfermagem (DE) mais incidentes foram ansiedade e medo, ambos referente ao aspecto psicológico. Por sua vez para o grupo nomeado Programa de Reabilitação Cardiovascular Supervisionado, teve três DE: dispneia, edema e pressão arterial alterada, mas estes não alcançaram uma frequência elevada entre os participantes.

No tocante aos resultados finais do estudo, foi evidenciado uma melhora quanto a presença dos DE encontrados no período pré e pós-intervenção. A Tabela 2 mostra o quantitativo de pacientes, de forma sucinta, que continham os diagnósticos antes e após as intervenções de enfermagem aplicadas mediante ao uso da TMA-Enf RCV.

Tabela 2. Resultados obtidos após a aplicação da TMA-Enf RCV (n=16).

\begin{tabular}{lcc}
\multicolumn{1}{c}{ Diagnósticos de Enfermagem } & Participantes (n) \\
\hline Ansiedade & Pré-intervenção & Pós-intervenção \\
Medo & 5 & 3 \\
Intolerância à atividade & 5 & 2 \\
Fadiga & 6 & 2 \\
Sono e repouso prejudicado & 4 & 3 \\
Angústia espiritual & 2 & 2 \\
Desesperança & 2 & -- \\
Autocuidado prejudicado & 4 & 1 \\
Desconforto & 1 & 3 \\
Atitude em relação ao manejo da medicação & 2 & -- \\
conflituosa & & Não foi possível avaliar \\
Baixa autoestima situacional & 3 & 1 \\
Edema & 3 & 1 \\
Dispneia & 3 & 2 \\
Pressão arterial alterada & 1 & 1 \\
\hline
\end{tabular}

Fonte: Elaborado pelos autores.

\section{Discussão}

A utilização da TMA Enf-RCV guiando as etapas do PE, possibilitou o desenvolvimento de ações específicas voltadas para a melhora do quadro clínico do paciente de acordo com as suas necessidades, as intervenções aplicadas foram de grande valia tanto para o início, quanto para o seguimento do processo de reabilitação cardiovascular dos participantes.

\subsection{Plano de cuidados implementados em pacientes com insuficiência cardíaca com foco na reabilitação cardiovascular}

A fim de melhor facilitar a compreensão, denominou-se como P seguido pelo numeral, a identificação dos pacientes participantes da pesquisa. 
O diagnóstico mais incidente entre os participantes foi Ansiedade (P1; P2; P3; P4; P9; P10; P11, P13 e P16) este por sua vez, pode ser definido como estado ou condição emocional debilitante e complexa que produz uma resposta interna (cognitiva) e externa (física) com potencial de alterar a qualidade de vida do ser acometido (Kindermann et al., 2019).

Vale ressaltar que os transtornos de humor, que englobam ansiedade e depressão, são frequentemente negligenciados na prática clínica, sendo muitas vezes o foco maior em diagnósticos relacionados à fisiologia humana. A identificação em pacientes com IC é ainda mais desafiadora, tendo em vista a sobreposição de diversos sintomas, como fadiga, perda ponderal e distúrbios do sono (Gomes \& Bocchi, 2020).

As intervenções de enfermagem (IE) utilizadas tiveram foco em aliviar o nível de tensão dos participantes, buscando promover um ambiente mais confortável, acolhedor e menos entediante. Foi feito uso de palavras cruzadas, dominó e dama, estas ações melhoraram na relação interpessoal dos participantes com os pesquisadores, bem como com outros pacientes da unidade. Realizou-se, também, conversas pontuais e específicas sobre o funcionamento da unidade e sua característica clínica, com os pacientes e familiares, objetivando minimizar a angústia.

A Teoria em discussão descreve sobre a importância de trabalhar os aspectos, social e psicológico no âmbito da reabilitação cardiovascular, sendo de fundamental importância para a qualidade de vida e todo o processo de restabelecimento das condições normais do cotidiano. Também afirma que o enfermeiro, quando baseado na TMA Enf-RCV, pode estimular os pacientes a viver em um ambiente reabilitador que propicie melhoria a saúde (Farias, 2018).

De acordo com os pressupostos da TMA-Enf RCV, as pessoas são seres que podem vivenciar um evento cardiovascular e necessitam de cuidados reabilitadores para experienciarem o processo de $\mathrm{RCV}$, logo é importante o mínimo conhecimento sobre o seu adoecimento cardíaco bem como do que é o processo de reabilitação, e como este último pode o ajudar na sua adaptação a nova forma de viver (Farias, 2018).

O DE medo (P1, P4, P5, P7 e P9) pode ser definido como resposta a uma ameaça percebida que é conscientemente reconhecida como um perigo, podendo também estar relacionado com a falta de apoio familiar/social ou a um estímulo externo que venha a desencadear um mecanismo liberador inato (Herdman et al., 2021).

Os pacientes entrevistados, ao serem questionados sobre como que se sentiam, apresentaram apreensão e dificuldades para se expressarem. Nessa perspectiva, as intervenções implementadas, buscaram auxiliar os mesmos a identificar situações que provocavam medo e resolvê-las com uma abordagem educativa. Dessa forma, eles apresentaram mais domínio sobre o seu quadro clínico, adquiriram mais conhecimento e diminuíram o nervosismo diante da chegada dos profissionais na enfermaria.

A necessidade de atentar para o lado psicológico frente ao adoecimento cardíaco é essencial para o sucesso do processo de reabilitação, uma vez que este aspecto pode limitar a esperança e o desejo da sequência, do ser acometido, para com a terapêutica. O coração carrega consigo um simbolismo histórico, uma vez que é tido como sede das paixões e sentimentos. Assim, quando esse órgão adoece, a sensação de impotência desencadeia uma séria de respostas psíquicas que ocasiona desequilíbrios psicológicos e de enfretamento, causando medo, ansiedade, desesperança e, muitas vezes, luto antecipado (Goulart \& Silveira, 2020).

Outro ponto bastante relevante para o processo de RCV em pacientes com IC é aspecto físico, estes, historicamente apresentam pouca tolerância à atividade física, dependendo do grau de comprometimento, experimentam facilmente quadros de dispneia e fadiga, gerando efeitos negativos tanto na prática de exercício físico como nas atividades do cotidiano, levando a uma grande redução da qualidade de vida destes indivíduos (Fernandes, 2020).

Após analisar os achados da pesquisa, evidenciou-se que o DE intolerância à atividade, juntamente com fadiga, teve notável significância, chegando a um total de 10 participantes $(62,5 \%)$ com pelo menos uma dessas duas condições. A intolerância à atividade (P1, P2, P5, P11 e P16) pode ser definida como energia fisiológica ou psicológica insuficiente para suportar ou completar as atividades básicas requeridas ou desejadas, podendo limitar as atividades de vida diárias e qualidade 
de vida que pode estar relacionada a um desequilíbrio entre a oferta e a demanda de oxigênio (Herdman et al., 2021; Roy, 2014). As intervenções para estes diagnósticos foram as orientação sobre a prática de exercícios passivos, como movimentos no próprio leito/alongamentos, e os exercícios ativos, mais precisamente a prática da deambulação, além de esclarecer as eventuais dúvidas sobre o adoecimento.

Por sua vez, o DE fadiga (P4, P5, P6, P8, P10 e P14) ) é definido como uma sensação física opressiva e sustentada de exaustão com capacidade diminuída para realizar trabalho físico e mental das atividades laborais e de vida diária no nível habitual, geralmente com sintomas de cansaço, que não é aliviado com o emprego de estratégias usuais de restauração da energia, como o repouso (Nóbrega, 2018; Pereira et al., 2016). As IE implementadas para o DE supracitado foram focadas em minimizar a fadiga, assim buscou-se identificar os fatores predisponente, explicar para o paciente e acompanhante as possíveis causas da fadiga, orientar técnicas de relaxamento a partir da respiração, estimular exercícios passivos no leito, como aumento da amplitude dos membros e alongamentos, e buscar oferecer o máximo de conforto possível aos participantes.

Outro ponto identificado, que necessitou de intervenções para o processo de reabilitação, foi a religiosidade. Após o primeiro contato com os participantes, notou-se que P8 e P15 apresentaram alterações nesse aspecto, uma vez que estavam aflitos e justificaram suas atuais situações clínicas considerando o fator religião. Em uma das conversas, P8 indagou "por que Deus deixa isso acontecer comigo?". Por sua vez, P15 afirmou que seu sofrimento estava relacionado ao distanciamento da igreja “...tudo isso que tá acontecendo comigo é porque eu parei de frequentar a casa do Pai, quando eu ia constantemente, não sentia, nem passava por todos esses problemas".

Nessa perspectiva, para esses dois participantes, estabeleceu-se o DE angústia espiritual que pode ser definido como a capacidade prejudicada de integrar significado e propósito à vida, falta de conexão consigo, com Deus ou com os outros ao seu redor, expressando isolamento, sofrimento espiritual, mudança de comportamento, falta de esperança, sentimento de culpa, sensação de abandono, raiva de Deus ou outra força maior (Nóbrega, 2018; Simão et al., 2015).

Ainda relacionado ao aspecto espiritual, o DE baixa autoestima situacional (P4, P11 e P15) e desesperança (P1 e P7) também foram identificados, o que ocasionou algumas dificuldades na abordagem frente a sequência da pesquisa, pois os referidos participantes se sentiam indispostos. No entanto as abordagens realizadas permitiram uma melhora e a sequência do processo. As intervenções implementadas objetivaram facilitar a capacidade deles em comunicar suas necessidades relacionadas a demandas espirituais, observar sentimentos de tristeza, irritabilidade, medo, ansiedade e solidão, buscando oferecer apoio, e ensinar técnicas de relaxamento, onde o pesquisador levou músicas e incentivou a leitura da bíblia aos pacientes.

Portanto, por meio da interação com os participantes, notou-se que os aspectos físico, psicológico, social e espiritual, estão constantemente presentes e alterados nos seres com o diagnóstico de IC. Nesse sentido, o uso da TMA Enf-RCV se torna essencial para o cuidado dessa clientela, uma vez que oferece uma forma de cuidar com estrutura conceitual e prática, voltada para o processo de reabilitação cardiovascular.

\subsection{Avaliação da aplicação do plano de cuidados elaborado a partir da TMA Enf-RCV}

Após aplicação das intervenções, foi realizado um último encontro com os participantes a fim de analisar se as ações de cuidado, baseados no processo de reabilitação da TMA Enf-RCV tinham sido eficazes. Evidenciou-se que em grande parte dos participantes, aproximadamente $75 \%$, os resultados foram positivos.

A avaliação da presença dos diagnósticos antes e após as intervenções de enfermagem foram estabelecidos a partir da melhora do quadro clínico dos pacientes após as intervenções, foi avaliado, entre outros pontos, a frequência cardíaca, frequência respiratória, diminuição da inquietação, dos sinais de tristeza, fáceis de dor, melhora da relação interpessoal, melhor aceitação do diagnóstico e aumento da autoestima. Dessa forma, avaliou-se os participantes de forma individualizada, 
analisando cada aspecto da TMA Enf-RCV que foi estudo, sendo eles o psicológico, físico, social e espiritual. Devido à proximidade dos achados, optou-se por descrevê-los em dois grupos.

Os aspectos psicológico e espiritual foram os que obtiveram melhores resultados. O nível de ansiedade e medo diminuiu significantemente entre os participantes da pesquisa, o que comprovou que o ganho de conhecimento e informações sobre o quadro clínico, por parte dos pacientes, ajuda na minimização da angústia, no sentimento de impotência e medo. A oportunidade de expressar suas ideias e sentimentos foi essencial tanto para o desenvolvimento do vínculo, como para a evolução própria dos pacientes no quesito espiritualidade.

O apoio por meio da incorporação de intervenções de saúde, foi dado com uma perspectiva holística, para melhorar a reabilitação e ajudá-los a se adaptar ao novo estado de ser portador de insuficiência cardíaca, o que facilitou ao lidar melhor com a doença e reduzir as complicações associadas. Com isso, a atuação do profissional enfermeiro, frente ao processo de reabilitação cardiovascular dos pacientes tem papel de provisão, promoção, manutenção e restauração do conforto, seja ele psicológico ou físico, tendo em vista que conforto é um ponto esperado pelos pacientes e familiares em todo o processo de tratamento, além de ser uma meta da enfermagem (Cardoso et al., 2020). Por conseguinte, o aspecto físico e o social melhoraram significantemente, mesmo com todas as dificuldades encontradas durante a pesquisa. $\mathrm{O}$ uso de técnicas baseadas em exercícios passivos, como aumento da amplitude dos membros e alongamentos, foi essencial para tal eficácia.

Pelo fato de estar em um contexto hospitalar, a atenção para o aspecto físico focou em auxiliar o paciente a retomar gradualmente as atividades diárias e no controle do processo da doença, com mudanças duradouras de hábitos e comportamentos. Dessa forma, as ações de cuidados realizadas, permitiram que os participantes conhecessem algumas estratégias capazes de melhorar o seu processo de reabilitação, bem como sua qualidade de vida, intra e pós-hospitalar.

O diagnóstico atitude em relação ao manejo da medicação conflituosa foi identificado em dois participantes, justificado pelo fato de não conseguirem tomar os medicamentos da forma adequada quando estavam em casa. Por esse motivo, mesmo após a administração das intervenções a fim de promover uma melhora dessa condição, não foi possível avaliar se houve sucesso, uma vez que o contato do pesquisador com os participantes ocorreu apenas em ambiente hospitalar.

O fator social, embora não tenha tido diagnósticos específicos elencados para ele, foi um ponto identificado com desequilíbrio entre os participantes da pesquisa. Para a melhora dessa condição, buscou-se desenvolver algumas atividades socioeducativas com os mesmos e seus respectivos familiares, considerando que a interação social é importante para a sequência da terapêutica. Dessa forma, foi estimulado o uso de alguns jogos que permitissem a interação entre pacientes e acompanhantes além do uso do celular, para comunicação com amigos e familiares.

As principais dificuldades encontradas no presente estudo se deram devido à atual pandemia causada pelo Sars-Cov2, limitando os encontros com os pacientes e o processo de aproximação, além de impossibilitar a realização de algumas intervenções e o acesso a determinadas enfermarias. O pequeno tamanho amostral foi uma limitação ocasionada por dois fatores: a dificuldade de recrutamento de participantes causada pelas restrições estabelecidas em decorrência da pandemia e a existência de condições físicas que impossibilitaram a inclusão de alguns sujeitos na pesquisa.

Todavia, o objetivo principal da presente investigação foi alcançado, uma vez que se discutiu a utilização da TMA Enf-RCV, a partir da implementação de intervenções de enfermagem, contribuindo para o processo de reabilitação cardiovascular de pacientes com IC, destacando sua importância e relevância clínica em todo o processo reabilitativo do ser em relação aos aspectos físico, social, psicológico e espiritual.

\section{Conclusão}

No presente estudo, foi possível aplicar intervenções de enfermagem diante de diagnósticos de enfermagem identificados em pacientes com insuficiência cardíaca, internados em uma unidade hospitalar de referência do estado do Ceará, 
com base em um subconjunto terminológico da CIPE® a partir da utilização da TMA Enf-RCV. Ao final, foram identificados 14 DE. Os DE mais incidentes foram fadiga e intolerância à atividade, ambos relacionados ao conceito de Terapia Baseada no Exercício Físico. Conclui-se que o cuidado direcionado ao paciente com IC exige dos profissionais uma visão qualificada e atenciosa sobre o processo de reabilitação, em especial para os aspectos físico e psicológico.

O uso da TMA Enf-RCV possibilitou uma abordagem qualificada e favoreceu um cuidado científico e eficiente frente às necessidades identificadas quanto ao processo de reabilitação, os auxiliando a se adaptarem à nova forma de viver $\mathrm{e}$ minimizando as chances de um novo evento cardíaco. Novos estudos devem ser desenvolvidos sobre a aplicabilidade prática das teorias de enfermagem, em especial a TMA-Enf RCV, que se mostrou eficiente na presente investigação.

\section{Referências}

Brown, R. A. (1964). Rehabilitation of patients with cardiovascular diseases. Report of a WHO expert committee. World Health Organization Technical Report Series, 270, 3-46. https://apps.who.int/iris/bitstream/handle/10665/40577/WHO_TRS_270.pdf?sequence=1\&isAllowed=y

Cardoso, R. B., Souza, P. A., Caldas, C. P., \& Bitencourt, G. R. (2020) Diagnóstico de enfermagem em idosos hospitalizados à luz da teoria do conforto de Kolcaba. Revista de Enfermagem Referência, 5(4), e20066. http://doi.org/10.12707/RV20066

Conselho Federal de Enfermagem (2009). Resolução COFEN No 358/2009. Dispõe sobre a Sistematização da Assistência de Enfermagem e a implementação do Processo de Enfermagem em ambientes, públicos ou privados, em que ocorre o cuidado profissional de Enfermagem e dá outras providências. http://www.cofen.gov.br/resoluo-cofen-3582009_4384.html

Farias, M. S. (2018). Reabilitação cardiovascular: proposta de uma teoria de enfermagem de médio alcance. Dissertação de Mestrado, Universidade Estadual do Ceará, Fortaleza, CE, Brasil.

Farias, M. S., Silva, L. F., Brandão, M. A. G., Guedes, M. V. C., Pontes, K. M. A., \& Lopes, R. O. P. (2021). Medium reach theory for nursing in cardiovascular rehabilitation. Revista Brasileira de Enfermagem, 74(3), e20190718. http://doi.org/10.1590/0034-7167-2019-0718

Fernandes, M. A. F. (2020). Associação entre a atividade física e o estado clínico e nutricional de pacientes com insuficiência cardíaca. Trabalho de Investigação, Universidade do Porto, Porto, Portugal. https://repositorio-aberto.up.pt/bitstream/10216/127833/2/407996.pdf

Gomes, B. R., \& Bocchi, E. A. (2020). Qualidade de vida em insuficiência cardíaca: um objetivo importante no tratamento. Arquivos Brasileiros de Cardiologia, 114(1), 33-34. http://doi.org/10.36660/abc.20190741

Goulart, R. S., \& Silveira, B. B. (2020). O coração e as emoções: uma via de mão dupla entre o corpo e mente. Revista Mosaico, 11(2), 169-73. http://doi.org/10.21727/rm.v11i2.2343

Herdman, T. H., Kamitsuru, S., \& Lopes, C. T. (2021). Diagnósticos de Enfermagem da NANDA-I: definições e classificação 2021 -2023 (12a ed.). Artmed.

Kindermann, L., Traeberti, J., \& Nunes, R. D. (2019). Validação de uma escala de ansiedade para procedimentos diagnósticos pré-natais. Revista de Saúde Pública, 18(53), 1-10. http://doi.org/10.11606/S1518-8787.2019053000621

Leandro, T. A., Nunes, M. M., Teixeira, I. X., Lopes, M. V. O., Araújo, T. L., Lima, F. E. T., \& Silva, V. M. (2018). Desenvolvimento das teorias de médio alcance na enfermagem. Revista Brasileira de Enfermagem, 73(1), e20170893. http://doi.org/10.1590/0034-7167-2017-0893

Lima, M. F. G., Carvalho, J. C., Vasconcelos, E. M. R., Borba, A. K. O., Zimmermann, R. D., \& Costa, E. F. F. (2019) A importância da avaliação do letramento funcional em saúde no idoso: revisão integrativa. Revista Enfermagem Atual In Derme, 90(28), 1-7. http://doi.org/10.31011/reaid-2019-v.90-n.28art.537

Nóbrega, M. M. L. (2018). Nomenclatura de diagnósticos, resultados e intervenções de enfermagem para pacientes hospitalizados em unidades clínicas, utilizando a CIPE®. Ideia. https://www.researchgate.net/publication/328028145_EBOOK_Nomenclatura_de_diagnosticos_resultados _e_intervencoes_de_enfermagem_utilizando_a_CIPE

Pereira, J. M. V., Cavalcanti, A. C. D., Lopes, M. V. O., Silva, V. G., Souza, R. O., \& Gonçalves, L. C. (2016). Acurácia na inferência de diagnósticos de enfermagem de pacientes com insuficiência cardíaca. Revista Brasileira de Enfermagem, 68(4), 690-696. http://doi.org/10.1590/0034-7167.2015680417i

Poffo, M. R., Assis, A. V., Fracasso, M., Londero Filho, O. M., Alves, S. M. M., Bald, A. P., Schmitt, C. B., \& Alves Filho, N. R. (2017). Perfil dos pacientes internados por insuficiência cardíaca em hospital terciário. International Journal of Cardiovascular Sciences, 30(3), 189-198. http://doi.org/10.5935/23594802.20170044

Polit, D. F. (2019). Fundamentos de pesquisa em enfermagem: avaliação de evidências para a prática de enfermagem (7a ed.). Artmed.

Rolim, M. N. N. (2020). Subconjunto terminológico da CIPE® para o cuidado de enfermagem à pessoa com insuficiência cardíaca crônica. Dissertação de Mestrado, Universidade Regional do Cariri, Crato, CE, Brasil.

Roy, C. (2014). Generating middle range theory: from evidence to practice. Springer.

Simão, T. P., Chaves, E. C. L., \& Iunes, D. H. (2015). Angústia espiritual: a busca por novas evidências. Revista de Pesquisa Cuidado é Fundamental Online, 7(2), 2591-2602. http://doi.org/10.9789/2175-5361.2015.v7i2.2591-2602 
Research, Society and Development, v. 11, n. 3, e25011326579, 2022

(CC BY 4.0) | ISSN 2525-3409 | DOI: http://dx.doi.org/10.33448/rsd-v11i3.26579

Sociedade Brasileira de Cardiologia. Comitê Coordenador da Diretriz de Insuficiência Cardíaca (2018). Diretriz brasileira de insuficiência cardíaca crônica e aguda. Arquivos Brasileiros de Cardiologia, 111(3), 436-539. http://doi.org/10.5935/abc.20180190

Xavier, C. A., Figuereido, P. P., \& Canuso, L. D. S. (2020). Construção de tecnologia educativa digital para o autocuidado em insuficiência cardíaca. Research, Society and Development, 9(8), e929986406. http://doi.org/10.33448/rsd-v9i8.6406 